Cite this: Phys. Chem. Chem. Phys., 2011, 13, 6442-6451

\title{
Unusual decrease in conductivity upon hydration in acceptor doped, microcrystalline ceria
}

\author{
William C. Chueh, ${ }^{a}$ Chih-Kai Yang, ${ }^{a}$ Carol M. Garland, ${ }^{a}{ }^{\text {Wei Lai }}{ }^{b}$ and \\ Sossina M. Haile*a \\ Received 19th October 2010, Accepted 27th January 2011 \\ DOI: $10.1039 / \mathrm{c0cp02198a}$
}

The impact of hydration on the transport properties of microcrystalline $\mathrm{Sm}_{0.15} \mathrm{Ce}_{0.85} \mathrm{O}_{1.925}$ has been examined. Dense, polycrystalline samples were obtained by conventional ceramic processing and the grain boundary regions were found, by high resolution transmission electron microscopy, to be free of impurity phases. Impedance spectroscopy measurements were performed over the temperature range 250 to $650{ }^{\circ} \mathrm{C}$ under dry, $\mathrm{H}_{2} \mathrm{O}$-saturated, and $\mathrm{D}_{2} \mathrm{O}$-saturated synthetic air; and over the temperature range 575 to $650{ }^{\circ} \mathrm{C}$ under $\mathrm{H}_{2}-\mathrm{H}_{2} \mathrm{O}$ atmospheres. Under oxidizing conditions humidification by either $\mathrm{H}_{2} \mathrm{O}$ or $\mathrm{D}_{2} \mathrm{O}$ caused a substantial increase in the grain boundary resistivity, while leaving the bulk (or grain interior) properties unchanged. This unusual behavior, which was found to be both reversible and reproducible, is interpreted in terms of the space-charge model, which adequately explains all the features of the measured data. It is found that the space-charge potential increases by $5-7 \mathrm{mV}$ under humidification, in turn, exacerbating oxygen vacancy depletion in the space-charge regions and leading to the observed reduction in grain boundary conductivity. It is proposed that the heightened space-charge potential reflects a change in the relative energetics of vacancy creation in the bulk and at the grain boundary interfaces as a result of water uptake into the grain boundary core. Negligible bulk water uptake is detected under both oxidizing and reducing conditions.

\section{Introduction}

Ceria-based oxides have been investigated extensively for application in a range of energy technologies. These include heterogeneous catalysis, where they serve as active supports, ${ }^{1}$ fuel cells, where they can serve as either electrolyte (due to fast oxygen-ion conduction) $)^{2,3}$ or anode electrocatalyst (due to mixed conduction), ${ }^{4-6}$ and, more recently, as oxygen storage materials for solar thermochemical dissociation of water ${ }^{7,8}$ and carbon dioxide 7 to fuels. In all of these processes, water vapor is present at significant pressures and hence may plausibly impact overall device operation. Proton solubility in pure and doped ceria and zirconia has been investigated by secondary ion mass spectrometry ${ }^{9,10}$ and shown to be higher in polycrystalline samples than single crystals. ${ }^{9}$ In addition, water uptake in the grain boundary regions of nanostructured doped ceria ${ }^{11-15}$ and zirconia ${ }^{14,16,17}$ has been shown to dominate ionic transport at temperatures below $\sim 150{ }^{\circ} \mathrm{C}$. This intriguing result motivates a quantitative exploration of

\footnotetext{
${ }^{a}$ Materials Science, California Institute of Technology,

1200 E. California Blvd. Pasadena, California 91125.

E-mail:smhaile@caltech.edu

${ }^{b}$ Department of Chemical Engineering and Materials Science, Michigan State University, 2527 Engineering Building, East Lansing, Michigan 48824
}

the limits of the influence of water on the properties, particularly grain boundary properties, of doped ceria with dopant concentrations and microstructure relevant to applications as solid electrolytes and electrodes. Furthermore, because changes in water vapor pressure are often employed to vary gas-phase oxygen chemical activity in the study of electrolyte and mixed conducting materials, it is essential to establish whether observed changes in properties are due solely to induced variations in oxygen partial pressure or possibly reflect bulk-phase interactions with water vapor.

\section{Background}

In acceptor-doped ceria examined under dry conditions ranging from oxidizing (i.e. air) to moderately reducing (i.e. $p_{\mathrm{O}_{2}} \sim 10^{-25} \mathrm{~atm}$ at $650{ }^{\circ} \mathrm{C}$ ), the majority carriers are oxygen vacancies, generated as a consequence of the doping and requirements of charge neutrality in the bulk. Specifically, the bulk vacancy concentration is given by

$$
2\left[\mathrm{~V}_{\mathrm{O}}^{\bullet \bullet}\right]_{\infty}^{\mathrm{dry}}=\left[\mathrm{Sm}_{\mathrm{Ce}}^{\prime}\right]_{\infty}
$$

where Kröger-Vink notation has been employed, square brackets indicate concentration, the subscript $\infty$ indicates the bulk (located far from any interface), and the superscript 'dry' indicates a system without an appreciable proton concentration. 
The trivalent samarium dopant (15 at.\%) is taken to be immobile at the experimental temperatures; the electron (small polaron) concentration and conductivity, computed to be seven and six orders of magnitude smaller, respectively, than the oxygen ion vacancy concentration and conductivity at the oxidizing conditions examined here, ${ }^{5}$ are taken to be negligible.

Under humidification one can, in principle, consider proton uptake in a manner analogous to that which occurs in proton-conducting perovskites such as doped barium zirconates and cerates. ${ }^{18,19}$ In those materials, water incorporation is described by the reaction

$$
\mathrm{H}_{2} \mathrm{O}(\mathrm{g})+\mathrm{V}_{\mathrm{O}}^{\bullet \bullet}+\mathrm{O}_{\mathrm{O}}^{\times} \rightarrow 2 \mathrm{OH}_{\mathrm{O}}^{\bullet}
$$

where the incorporated protons, residing within the electron cloud of oxygen ions, are equivalent to hydroxyl groups.

If the proton concentration becomes appreciable, the electroneutrality approximation must be modified to reflect the presence of these species, becoming

$$
2\left[\mathrm{~V}_{\mathrm{O}}^{\bullet \bullet}\right]_{\infty}^{\text {hum }}+\left[\mathrm{OH}_{\mathrm{O}}^{\bullet}\right]_{\infty}^{\text {hum }}=\left[\mathrm{Sm}_{\mathrm{Ce}}^{\prime}\right]_{\infty}
$$

where the superscript 'hum' indicates a humidified atmosphere. The total bulk conductivity under such conditions becomes:

$$
\sigma_{\infty, \text { tot }}=\sigma_{\infty, \text { ion }}^{\text {hum }}+\sigma_{\infty, \text { pon }}^{\text {hum }}
$$

where $\sigma_{\infty \text {,ion }}^{\text {hum }}$ and $\sigma_{\infty \text {,pon }}^{\text {hum }}$ are the ionic and protonic conductivity, respectively.

Under reducing atmospheres (obtained here by mixing $\mathrm{H}_{2}$ and $\mathrm{H}_{2} \mathrm{O}$ ), cerium undergoes partial reduction from the $4+$ to $3+$ oxidation state, giving rise to mixed ionic and electronic conduction. ${ }^{20}$ The formation of localized electrons and intrinsic oxygen vacancies can be expressed as

$$
\mathrm{O}_{\mathrm{O}}^{\times} \leftrightarrow \frac{1}{2} \mathrm{O}_{2}(\mathrm{~g})+\mathrm{V}_{\mathrm{O}}^{\bullet \bullet}+2 \mathrm{e}^{\prime}
$$

Under the most extreme conditions examined here, the electron concentration remains below 30\% (electrical charge) that of oxygen vacancies and thus polarons need not be introduced into the charge neutrality condition (eqn (3)). ${ }^{5,21}$ Nevertheless, their high mobility renders them significant as charge carriers such that the total bulk conductivity under moderately reducing conditions is given by:

$$
\sigma_{\infty, \text { tot }}=\sigma_{\infty, \text { ion }}^{\text {hum }}+\sigma_{\infty, \text { pon }}^{\text {hum }}+\sigma_{\infty, \text { eon }}^{\text {hum }}
$$

where $\sigma_{\infty, \text { eon }}^{\text {hum }}$ is the electronic conductivity, and, again, the superscript 'hum' indicates a humidified atmosphere. In the limit that proton concentration and conductivity are negligible, it is readily shown that the total conductivity can be written as:

$$
\sigma_{\infty, \text { tot }}=\sigma_{\infty, \text { ion }}^{\mathrm{dry}}+\sigma_{\infty, \text { eon }}^{0} p_{\mathrm{O}_{2}}^{-1 / 4}
$$

where $\sigma_{\infty}^{\text {dry }}$,ion is the oxygen vacancy conductivity measured under dry, oxidizing atmosphere, and $\sigma_{\infty, \text { eon }}^{0}$ is the $p_{\mathrm{O}_{2}}{ }^{-}$ independent term in the electronic conductivity.

\section{Experimental section}

\section{Sample preparation and physical characterization}

Commercial powder of $15 \%$ samarium-doped ceria $\left(\mathrm{Sm}_{0.15} \mathrm{Ce}_{0.85} \mathrm{O}_{1.925}\right.$, or SDC, Nextech Materials) was uniaxially compacted (280 MPa for $2 \mathrm{~min}$ ) into a disc and sintered at
$1350{ }^{\circ} \mathrm{C}$ for $5 \mathrm{~h}$ in an alumina tube furnace dedicated to the processing of SDC. These sintering conditions have been found to yield a satisfactory balance between grain size and impurity segregation to the grain boundaries. ${ }^{22}$ The sintered pellet $(13.0 \mathrm{~mm}$ in diameter and $0.62 \mathrm{~mm}$ in thickness, density $\sim 95 \%$ of theoretical) was polished aggressively to remove any species that might have segregated to the surface during sintering. For electrical measurements, platinum paste (BASF 6082) was applied to each side of the pellet and fired at $900{ }^{\circ} \mathrm{C}$ for a total dwell time of $140 \mathrm{~min}$. Ag mesh pieces affixed to the electrodes with silver adhesive paste served as current collectors.

Electron microscopy was employed to study the microstructural features of the sintered sample (after electrochemical transport measurements were concluded). Scanning electron microscopy (SEM, Carl-Zeiss LEO 1550 VP) was used to determine the grain size distribution. The bulk and grain boundary microstructure and chemical composition of the sample were further investigated by transmission electron microscopy (TEM, FEI Tecnai F30UT) in conjunction with energy dispersive spectroscopy (EDS, Oxford INCA). The sample was wet-polished, mounted on a Mo grid, dimpled, and thinned using an Ar ion mill. Analysis was carried out at three locations of the cross-sectioned sample: near the two faces and near the middle of the SDC disc. All images were collected at an accelerating voltage of $300 \mathrm{kV}$.

$\mathrm{X}$-Ray diffraction (XRD) patterns were collected from sintered pellets using a Philips X'Pert Pro diffractometer ( $\mathrm{Cu}-\mathrm{K} \alpha$ radiation, $45 \mathrm{kV}, 40 \mathrm{~mA}$ ).

\section{Transport measurements}

Transport measurements were made using electrochemical impedance spectroscopy under controlled temperatures and atmospheres. Both the electrolytic and the mixed ionic and electronic conduction regimes were explored. The electroded sample was placed inside a continuous flow furnace to which gases were delivered via digital mass flow controllers, and a four-probe configuration was employed to minimize inductance effects from the wiring.

Measurements under oxidizing conditions (i.e. in the electrolytic regime) were performed in nominally dry, $\mathrm{H}_{2} \mathrm{O}$ humidified, and $\mathrm{D}_{2} \mathrm{O}$-humidified atmospheres. Dry, oxidizing conditions were obtained by delivering a mixture of $99.9995 \%$ pure $\mathrm{O}_{2}$ and $\mathrm{Ar}$, with the oxygen partial pressure held constant at $0.21 \mathrm{~atm}$, directly to the sample, whereas humidification was achieved by first passing the mixed gas through a bubbler placed inside a variable-temperature water bath. The $\mathrm{H}_{2} \mathrm{O}$ vapor pressure was varied in the range from $5 \times 10^{-4}$ to $1.3 \times 10^{-2}$ atm by varying the bath temperature from 11 to $51{ }^{\circ} \mathrm{C}$ and mixing with dry gas. Measurements under $\mathrm{D}_{2} \mathrm{O}$ were carried out at a partial pressure of 0.013 atm (using a bath temperature of $14{ }^{\circ} \mathrm{C}$ ). The sample temperature was varied between 250 and $650{ }^{\circ} \mathrm{C}$ in $50{ }^{\circ} \mathrm{C}$ increments. Due to the possibility of slow hydration and dehydration kinetics at lower temperatures, changes to the gas atmosphere were made at $650{ }^{\circ} \mathrm{C}$ and impedance spectra collected on cooling, after a dwell time of 1.5 to $8 \mathrm{~h}$ at each temperature. The atmosphere was cycled back and forth several times between the three 
conditions to examine both reproducibility and reversibility of hydration-induced changes in transport behavior.

Measurements under reducing conditions (i.e. in the mixed conduction regime) were performed under $\mathrm{H}_{2} \mathrm{O}$-humidified mixtures of hydrogen and argon, with the hydrogen and water vapor pressures varied independently. Oxygen partial pressures in the range of $\sim 10^{-21}$ to $10^{-28}$ atm were accessed. Isotope effects were not probed in this case. Due to slow oxygen ambipolar diffusion at low temperatures, experiments were limited to temperatures between 575 and $650{ }^{\circ} \mathrm{C}$ (in $25^{\circ} \mathrm{C}$ increments), at which equilibration could be achieved within a moderate period. Stabilization times ranged from 6 to $12 \mathrm{~h}$ depending on conditions. In all cases (both oxidizing and reducing), effluent humidity was monitored using a Rotronic Hygroflex capacitative sensor. The total flow rate was kept constant at $101 \mathrm{~cm}^{3} \mathrm{~min}^{-1}$ (STP), corresponding to a linear gas velocity of $0.7 \mathrm{~cm} \mathrm{~s}^{-1}$.

Impedance data were collected using a Solartron 1260A frequency response analyzer at frequencies from 1 or $10 \mathrm{MHz}$ to $1,0.01$ or $0.001 \mathrm{~Hz}$ and at a zero-bias perturbation amplitude of $50 \mathrm{mV}$. Data analysis was performed as follows. Under oxidizing conditions, regardless of humidification, the impedance spectra, as plotted in the Nyquist representation, had the form of multiple symmetric arcs. Accordingly, the data were represented in terms of an equivalent circuit with multiple (RQ) parallel subcircuits placed in series with one another and in series with additional resistor, $R$, and inductor, $L$, elements, as appropriate; here, the constant phase element $Q$ has impedance $Z_{Q}=\left[(j \omega)^{n} Y\right]^{-1}$, and the equivalent capacitance of any given $R Q$ sub-circuit is computed from $C_{\text {eq }}=Y^{1 / n} R^{1 / n-1}$. Under the high temperature reducing conditions, only a single arc with an asymmetric tear-drop shape, characteristic of mixed conductors with high total conductivity, ${ }^{4,5}$ was observed and a physical impedance model described previously ${ }^{5}$ used to obtain physical parameters. The high frequency intercept so determined was taken to be the sum of the grain boundary and bulk resistances, $R_{\text {tot }}$, with contributions from all mobile species. Non-linear least squares fitting was implemented using the software package ZView (Scribner Associates).

\section{Results}

\section{Materials characterization}

X-Ray powder diffraction measurements confirmed that the sintered SDC disc had a cubic fluorite structure (lattice constant $a=5.430 \AA$ A) with no detectable second-phases, both before and after electrochemical characterization (not shown). High resolution transmission electron micrographs revealed the grain boundaries to be free of intergranular phases at all locations examined, as shown in the representative image in Fig. 1. In no instance was a crystalline precipitate or an amorphous region observed, consistent with previous TEM studies of similarly prepared material from the same vendor. ${ }^{23}$ In contrast, some authors have reported that high grain boundary resistance in doped ceria is due to amorphous phases. $^{24,25}$ EDS analysis indicated the absence of detectable impurities throughout the sample. From the SEM images
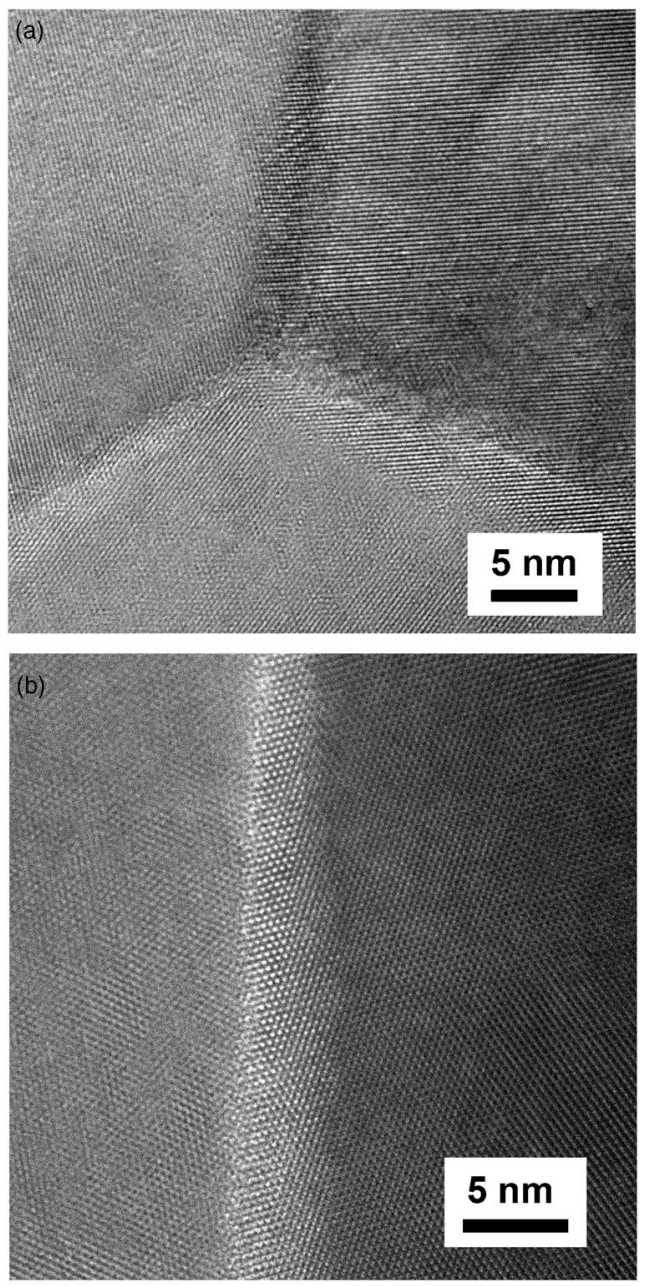

Fig. 1 Transmission electron micrographs of typical grain boundaries in polycrystalline samarium-doped ceria sintered at $1350{ }^{\circ} \mathrm{C}$ for $5 \mathrm{~h}$.

(not shown), the average grain size of the polished cross-section was estimated, using the mean-intercept method, to be $0.36 \mu \mathrm{m}$.

\section{Transport under oxidizing conditions}

At temperatures between 250 and $350{ }^{\circ} \mathrm{C}$ and all oxidizing atmospheres explored, the impedance spectra, presented in Nyquist plots, consisted of three distinct arcs (Fig. 2). The relative dielectric constant implied by the high frequency arc is $59\left(250{ }^{\circ} \mathrm{C}\right.$, dry) consistent with its assignment as the bulk response, ${ }^{22,24,26-28}$ reported values for doped ceria range from approximately 20 to $70 .^{22,29,30}$ The effective, relative dielectric constant implied by the mid-frequency arc is $1.8 \times 10^{4}$, a value that is consistent with grain boundary properties. In contrast, that of the low frequency (electrode) arc is $8.3 \times 10^{5}$. At temperatures above $350{ }^{\circ} \mathrm{C}$, the bulk arc became inaccessible, and finally above $450{ }^{\circ} \mathrm{C}$ only the electrode arc was accessible, typical behavior of polycrystalline materials with high conductivity and examined using instruments with standard frequency ranges. ${ }^{31}$ Accordingly, the bulk and grain boundary resistances are determined independently only at temperatures 


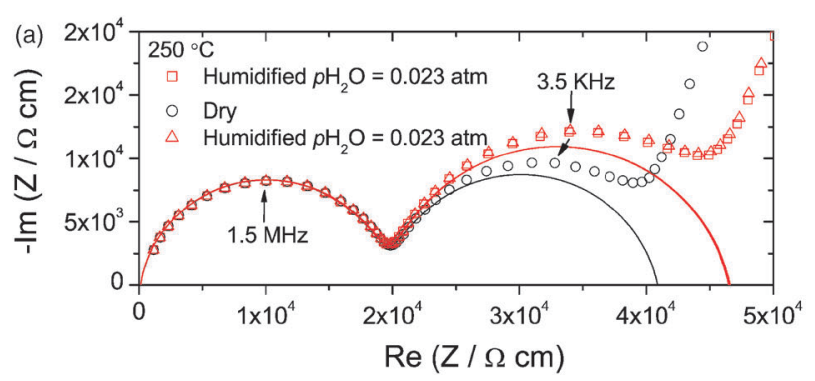

bulk, effective grain boundary and specific grain boundary conductivities, where the latter is obtained using the relationship given in eqn (9) below. In Fig. 3(b) we show the relative conductivities in $\mathrm{H}_{2} \mathrm{O}$-humidifed and dry atmospheres of the bulk, grain boundary and total response, and in Fig. 3(c) we present the relative conductivities of the bulk and grain boundaries regions under $\mathrm{H}_{2} \mathrm{O}$ and $\mathrm{D}_{2} \mathrm{O}$ humidification. At all temperatures at which the bulk conductivity could be
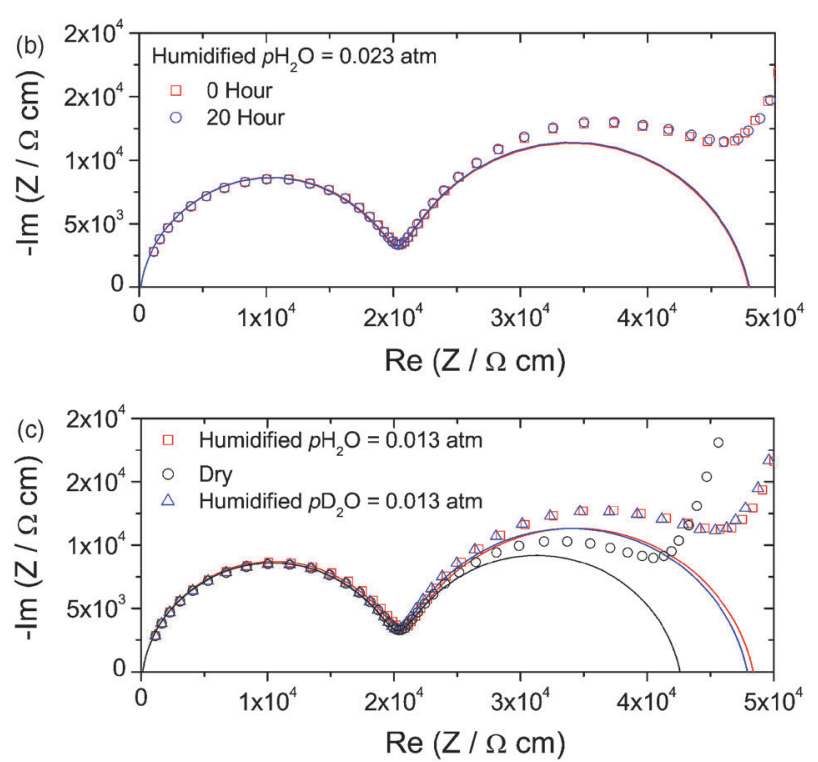

Fig. 2 Raw impedance spectra of $\mathrm{Sm}_{0.15} \mathrm{Ce}_{0.85} \mathrm{O}_{1.925}$ at at $250{ }^{\circ} \mathrm{C}$ (a) upon cycling in $\mathrm{H}_{2} \mathrm{O}$-saturated and dry atmospheres, (b) upon dwelling in $\mathrm{H}_{2} \mathrm{O}$-saturated atmosphere for $20 \mathrm{~h}$, and (c) upon cycling in $\mathrm{H}_{2} \mathrm{O}$-saturated, dry and $\mathrm{D}_{2} \mathrm{O}$-saturated atmospheres. Open points are the measured data and solid lines show the equivalent circuit fits, with the electrode response removed from the calculated spectra. Characteristic frequencies are essentially the same for all three plots and select frequencies are indicated in (a). $p_{\mathrm{O}_{2}}=0.21$ atm for all measurements.

of $450{ }^{\circ} \mathrm{C}$ and lower, and the associated capacitive responses for both processes only at temperatures of $350{ }^{\circ} \mathrm{C}$ and lower.

Even in the absence of any analysis, three characteristics of the impedance response are immediately apparent from Fig. 2. The first is that the grain boundary impedance increases on hydration, while the bulk impedance is unchanged; second, the behavior is, within measurement error, entirely reversible and reproducible; and, third, hydration under $\mathrm{H}_{2} \mathrm{O}$ and under $\mathrm{D}_{2} \mathrm{O}$ yield, within experimental error, identical transport characteristics. The reversibility is evident (Fig. 2(a)) from the superposition of the two sets of impedance data collected under $\mathrm{H}_{2} \mathrm{O}$ humidified atmospheres, where a measurement under dry conditions was performed between the two measurements. The impedance response remained invariant after dwelling for $20 \mathrm{~h}$ (Fig. 2(b)). The equivalence of $\mathrm{H}_{2} \mathrm{O}$ and $\mathrm{D}_{2} \mathrm{O}$ is evident (Fig. 2(c)) from the superposition of the two sets of impedance data collected under the differing atmospheres, where, again, a measurement under dry conditions was performed in between these data collections.

The temperature dependence of the transport properties under oxidizing conditions are summarized in Fig. 3 and Table 1. In Fig. 3(a) we show the Arrhenius behavior of the
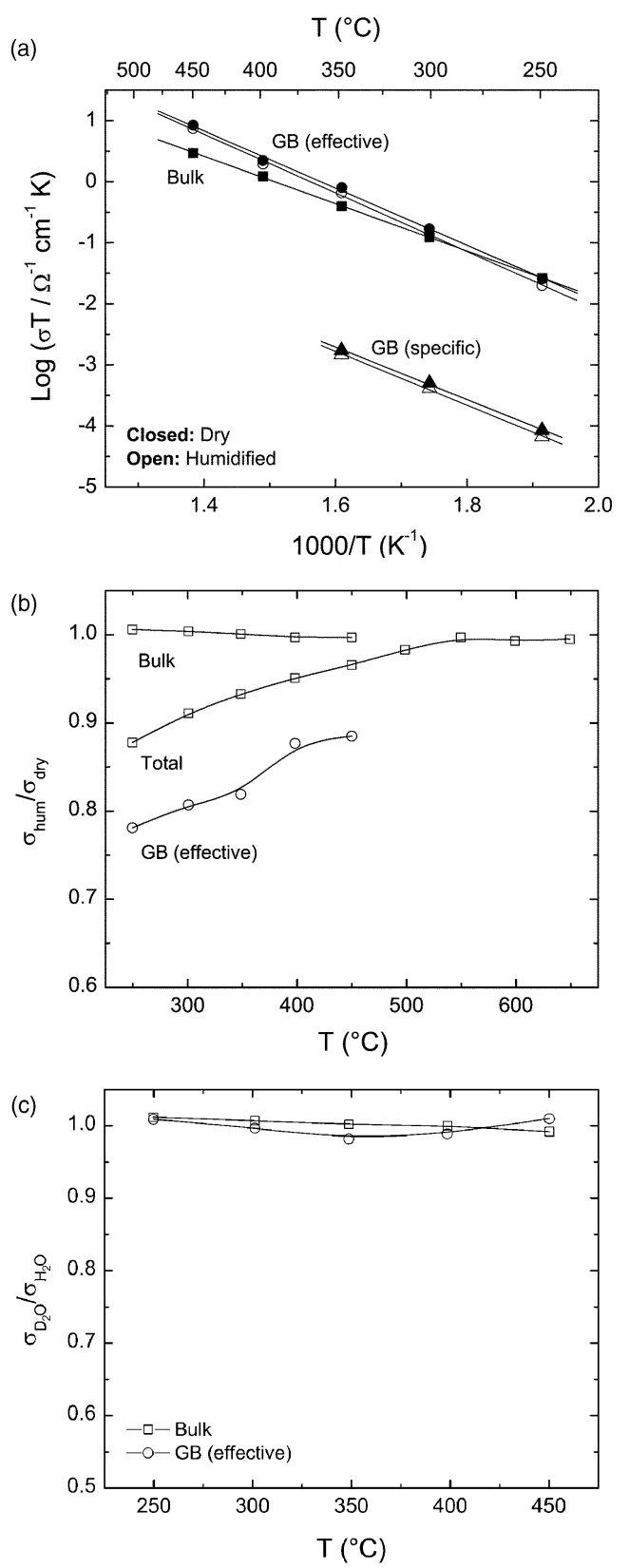

Fig. 3 Temperature-dependent transport properties of $\mathrm{Sm}_{0.15} \mathrm{Ce}_{0.85} \mathrm{O}_{1.925}$ under various conditions: (a) Arrhenius representation of bulk, effective and specific grain boundary conductivities under nominally dry and under humidified atmosphere $\left(p_{\mathrm{H}_{2} \mathrm{O}}=0.023 \mathrm{~atm}\right)$; (b) ratio of humidified ( $\left.p_{\mathrm{H}_{2} \mathrm{O}}=0.023 \mathrm{~atm}\right)$ to dry conductivities (total, bulk, and effective grain boundary) as functions of temperature; and (c) Ratio of $\mathrm{D}_{2} \mathrm{O}$-saturated to $\mathrm{H}_{2} \mathrm{O}$-saturated conductivities (bulk and effective grain boundary) at $0.013 \mathrm{~atm}$ vapor pressure. Lines in (b) and (c) are guides to the eye. $p_{\mathrm{O}_{2}}=0.21 \mathrm{~atm}$ for all measurements. 
Table 1 Apparent activation energy for total, bulk and grain boundary conductivities

\begin{tabular}{lll}
\hline & $\begin{array}{l}\text { Atmosphere } \\
\left(p_{\mathrm{O}_{2}}=0.21 \mathrm{~atm}\right)\end{array}$ & $\begin{array}{l}\text { Conductivity } \\
\text { activation } \\
\text { energy }(\mathrm{eV})\end{array}$ \\
\hline Bulk & Nominally dry & $0.77 \pm 0.01$ \\
& $p_{\mathrm{H}_{2} \mathrm{O}}=0.023 \mathrm{~atm}$ & $0.77 \pm 0.01$ \\
Grain boundary (effective) & Nominally dry & $0.93 \pm 0.02$ \\
& $p_{\mathrm{H}_{2} \mathrm{O}}=0.023 \mathrm{~atm}$ & $0.95 \pm 0.02$ \\
Grain boundary (specific) & Nominally dry & $0.85 \pm 0.03$ \\
& $p_{\mathrm{H}_{2} \mathrm{O}}=0.023 \mathrm{~atm}$ & $0.88 \pm 0.03$ \\
\hline
\end{tabular}

resolved, its value was essentially independent of hydration, Fig. 3(b). In contrast, the grain boundary conductivity, as previously evident from the raw impedance data, was, in all accessible cases, lower upon hydration than in a dry environment. Furthermore, humidification under $\mathrm{H}_{2} \mathrm{O}$ gave identical behavior as that under $\mathrm{D}_{2} \mathrm{O}$, Fig. 3(c), as was also already evident directly from the impedance spectra. With increasing temperature, the overall impact of hydration decreased. Specifically, whereas hydration to $p_{\mathrm{H}_{2} \mathrm{O}}=0.023$ atm lowered the grain boundary conductivity by $21.9 \%$ at $250{ }^{\circ} \mathrm{C}$, the decrease was only $11.5 \%$ at $450{ }^{\circ} \mathrm{C}$. Furthermore, the decrease in conductivity induced by humidification was found, specifically, for lower temperature measurements, to be dependent on the water partial pressure (apparently asymptotically), Fig. 4, directly demonstrating the influence of $\mathrm{H}_{2} \mathrm{O}$ chemical activity on the transport properties in SDC. The total conductivity, which reflects the behavior of both bulk and grain boundary conductivities, was similarly found to decrease significantly upon humidification at low temperatures (Fig. 3(b)) and with increasing $p_{\mathrm{H}_{2} \mathrm{O}}$, with limited sensitivity to humidity at the highest temperatures. At $500{ }^{\circ} \mathrm{C}$ and higher, the decrease in total conductivity upon hydration, by either $\mathrm{H}_{2} \mathrm{O}$ or $\mathrm{D}_{2} \mathrm{O}$, was less than $1 \%$.

\section{Transport under reducing conditions}

Typical impedance spectra obtained under high temperature, reducing conditions are presented in Fig. 5. It is immediately evident that for a given oxygen partial pressure and temperature $\left(p_{\mathrm{O}_{2}}=9 \times 10^{-26} \mathrm{~atm}, 600{ }^{\circ} \mathrm{C}\right.$ for the example shown) changes in the water vapor pressure have minimal impact on the

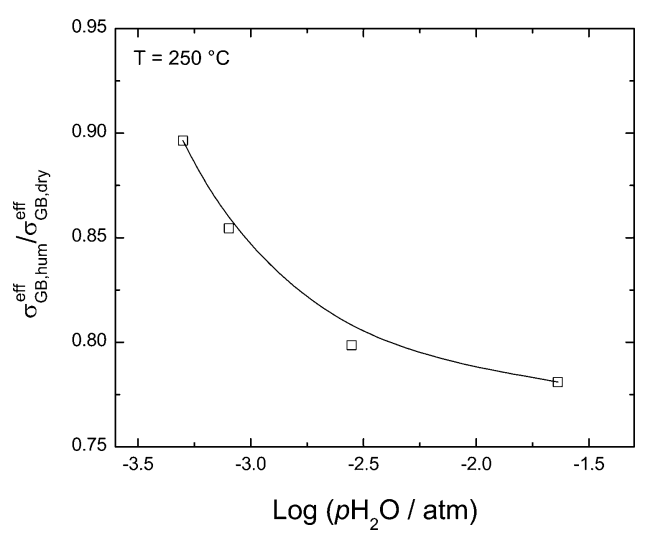

Fig. 4 Dependence of the effective grain boundary conductivity on water vapor pressure $\left(p_{\mathrm{O}_{2}}=0.21 \mathrm{~atm}\right)$. Line is a guide to the eye. electrochemical impedance behavior. Not only is the high frequency intercept, which reflects the sum of the grain boundary and bulk resistances, $R_{\text {tot }}$, unchanged, none of the features of the arc are impacted by the method of attaining the given oxygen chemical activity. Exploring the implication of this result on the electrochemical reaction behavior is beyond the scope of this work, and a full analysis will be presented elsewhere. Returning to $R_{\text {tot }}$, if one takes the grain boundary resistive behavior under reducing conditions to be adequately approximated by that obtained under oxidizing conditions, it is possible to extrapolate the results of Fig. 3(a) to high temperature and estimate the contribution of grain boundaries to $R_{\text {tot }}$ at any arbitrary temperature. Such an analysis indicates that at $575{ }^{\circ} \mathrm{C}$ the bulk ionic resistance contribution is $\sim 3.7$ times greater than the grain boundary ionic resistance contribution, with the ratio increasing at higher temperatures. Accordingly, $R_{\text {tot }}$ is taken here as the bulk resistance, without correction for the minor, grain boundary contributions.

In principle, $R_{\text {tot }}$ reflects conduction due to mixed ionic, electronic and protonic transport. Where proton transport does not play a role, eqn (7), it is apparent that $\sigma_{\infty \text {,tot }}-\sigma_{\infty \text {,ion (the }}^{\text {dry }}$ former term obtained from $R_{\text {tot }}$ and the latter from measurements under dry, oxidizing conditions) must correspond to the electronic conductivity. Thus, this quantity will be independent of atmospheric $\mathrm{H}_{2} \mathrm{O}$ vapor pressure, so long as the oxygen partial pressure is maintained. As shown in Fig. $6, \sigma_{\infty \text {,tot }}-\sigma_{\infty \text {,ion, }}^{\text {dry }}$, plotted as a function of oxygen partial pressure, is, indeed, independent of the absolute value of $p_{\mathrm{H}_{2} \mathrm{O}}$. That is, whether the oxygen partial pressure variation is achieved by varying $p_{\mathrm{H}_{2}}$ at fixed $p_{\mathrm{H}_{2} \mathrm{O}}$ or by varying $p_{\mathrm{H}_{2} \mathrm{O}}$ at fixed $p_{\mathrm{H}_{2}}$, the values coincide. Fitting $\sigma_{\infty \text {,tot }}-\sigma_{\infty \text {,ion }}^{\text {dry }}$ to a power-law dependence on oxygen partial pressure yields exponents ranging from -0.23 to -0.24 (when varying $p_{\mathrm{H}_{2} \mathrm{O}}$ ) and from -0.25 to -0.26 (when varying $p_{\mathrm{H}_{2}}$ ) which are close to the expected value of -0.25 (eqn (7)). Furthermore, the activation energies associated with this quantity $\left(2.42 \pm 0.05 \mathrm{eV}\right.$ for constant $p_{\mathrm{H}_{2}}$ and $2.36 \pm 0.05 \mathrm{eV}$ constant $p_{\mathrm{H}_{2} \mathrm{O}}$ ) are not only identical for the two sets of experiments, but also correspond within error to values reported in the literature for the electronic conductivity of SDC. ${ }^{4,5}$ This combination of observations, a -0.25 power law dependence on oxygen partial pressure and independence of properties on

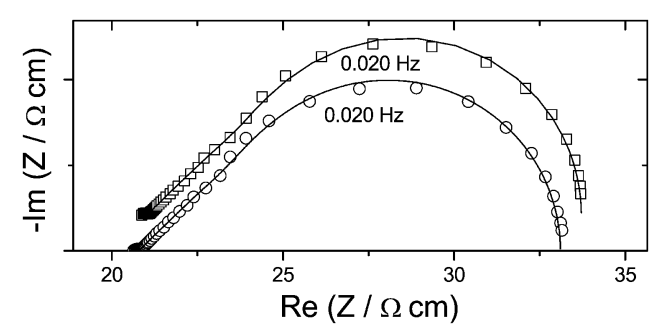

Fig. 5 Impedance spectra of $\mathrm{Sm}_{0.15} \mathrm{Ce}_{0.85} \mathrm{O}_{1.925}$ (with Pt electrodes) collected under reducing atmospheres at $600{ }^{\circ} \mathrm{C}$; (squares): $p_{\mathrm{H}_{2}}=0.16 \mathrm{~atm}$ and $p_{\mathrm{H}_{2} \mathrm{O}}=0.043 \mathrm{~atm}$; (circles): $p_{\mathrm{H}_{2}}=0.088 \mathrm{~atm}$ and $p_{\mathrm{H}_{2} \mathrm{O}}=0.023 \mathrm{~atm}$, with the two measurements having the same oxygen partial pressure, $9 \times 10^{-26} \mathrm{~atm}$. High frequency intercepts represent the total conductivity, which appear to attain the same value regardless of the hydrogen or water vapor partial pressure as long as the oxygen partial pressure is the same. For clarity the upper spectrum is shifted along the vertical axis. 

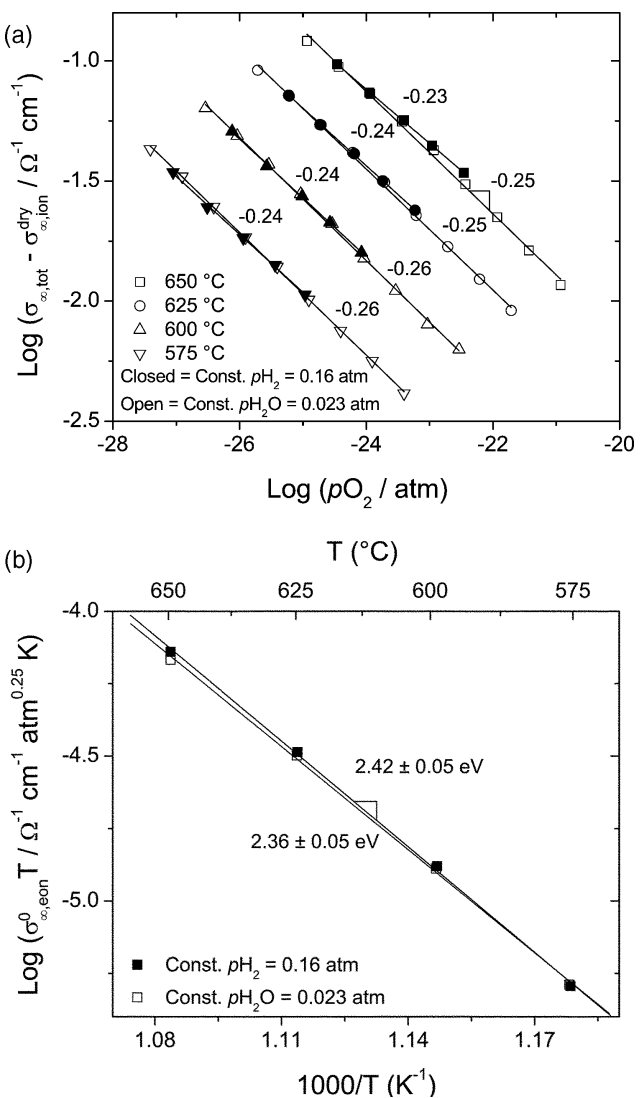

Fig. 6 SDC bulk properties obtained from analysis of the impedance data. Open and closed data points indicate measurements made under a constant $p_{\mathrm{H}_{2} \mathrm{O}}$ and constant $p_{\mathrm{H}_{2}}$, respectively. (a) Difference between the total conductivity and the ionic conductivity collected under dry air. (b) Taking the quantity plotted in (a) as the electronic conductivity (see text for details), the $p_{\mathrm{O}_{2}}$-independent term in the electronic conductivity $\left(\sigma_{\infty \text {, eon }}^{0}\right.$ in $\left.\sigma_{\infty, \text { eon }}=\sigma_{\infty, \text { eon }}^{0} p_{\mathrm{O}_{2}}^{-1 / 4}\right)$ is determined from each conductivity isotherm and plotted in the Arrhenius form.

water partial pressure for a given oxygen partial pressure, implies that the quantity $\sigma_{\infty}$,tot $-\sigma_{\infty \text {,ion }}^{\text {dry }}$ is the electronic conductivity and, that, under reducing atmospheres at moderate temperatures of 575 to $650{ }^{\circ} \mathrm{C}$, both proton incorporation and conductivity are negligible. Because the concentration of oxygen vacancies (sites required for water absorption, eqn (2)) is approximately identical in the oxidizing and reducing conditions, this result is consistent with the above observation that proton conductivity is insignificant under dry, oxidizing conditions at the same temperatures (Fig. 3(b)).

\section{Analysis and discussion}

In the overwhelming majority of cases, hydration of oxides leads to increases in conductivity due to the motion of incorporated hydroxyl or protonic species. ${ }^{32}$ The same is also true for grain boundaries, whereby proton incorporation into the grain boundary core leads to enhanced conductivity. ${ }^{31,33}$ The observation here of a decrease in grain boundary conductivity upon hydration motivates an analysis that does not directly involve migration of such species in the grain boundary core. Specifically, we explore whether space-charge effects, analyzed in terms of the combined brick layer and space-charge model, can explain the observed behavior. The analysis is expressly carried out for the measurements performed at lower temperatures and under oxidizing conditions, at which the grain boundary and bulk impedance responses were resolved separately.

In the brick layer model ${ }^{34}$ a random polycrystalline material is approximated by a uniform microstructure constructed of identical cubes. Within the framework of this model, the observation of a mid-frequency arc due to grain boundaries immediately implies that transport through grain boundaries that are parallel to the direction of current flow can be neglected. ${ }^{31}$ The grain boundaries aligned perpendicular to the direction of current flow (i.e., in series with the grains) have an effective conductivity given by $\sigma_{\mathrm{GB}}^{\mathrm{eff}}=\sigma_{\mathrm{GB}}^{\mathrm{sp}} \frac{l}{\delta}$ where $\sigma_{\mathrm{GB}}^{\mathrm{sp}}$ is the intrinsic conductivity, $l$ is the mean grain size, $\delta$ is the mean grain boundary thickness, and the effective conductivity is obtained from the macroscopically measured resistance, $R$, and the macroscopic sample dimensions. The mean grain size is accessible from direct microstructural examination. In contrast, the grain boundary thickness is not easily observed, and even its definition depends on the source of the resistive behavior of the grain boundaries.

The space-charge model, ${ }^{28,34-36}$ which has been used in the literature to accurately describe the behavior of a variety of oxides $^{26-28,37-40}$ and doped ceria in particular, ${ }^{26}$ assigns the high impedance of grain boundaries to carrier depletion in a space-charge region about the grain boundary core of vanishing thickness and negligible direct contribution to the measured electrical properties. As a consequence of the local disruptions of chemical bonding at the core (reflected in the altered energetics of defect formation ${ }^{28,34-36,39,41}$ ) the grain boundary core carries a net charge and, accordingly, an electrical potential difference from the bulk. The potential difference between the core and bulk is defined as the space-charge potential, $\Delta \phi_{\mathrm{sc}}$, and the spatial extent of the space-charge layer, $\lambda_{\mathrm{sc}}$, is defined in terms of the position at which the potential difference approaches zero. Each grain boundary core is sandwiched between two space-charge layers. Thus, the total thickness of the space-charge region is $2 \lambda_{\mathrm{sc}}$.

The identical structural characteristics of the bulk and space-charge regions justifies an assumption that the dielectric properties of the two are the same (as well as spatiallyindependent). This assumption, in turn, immediately permits an evaluation of the grain boundary thickness, $\delta$, according to

$$
\delta=l \frac{C_{\mathrm{GI}}}{C_{\mathrm{GB}}}
$$

and the specific grain boundary resistivity, $\sigma_{\mathrm{GB}}^{\mathrm{sp}}$, according to

$$
\sigma_{\mathrm{GB}}^{\mathrm{sp}}=\sigma_{\mathrm{GB}}^{\mathrm{eff}} \frac{C_{\mathrm{GI}}}{C_{\mathrm{GB}}}=\frac{1}{R_{\mathrm{GB}}} \frac{C_{\mathrm{GI}}}{C_{\mathrm{GB}}} \frac{L}{A}
$$

where $C_{\mathrm{GI}}$ and $C_{\mathrm{GB}}$ are the measured bulk (or grain interior) and grain boundary capacitances, respectively, $L$ is the sample length and $A$ its area. Under the assumption that the core is reversible to charge transport, the grain boundary thickness obtained from eqn (8) is expected to correspond to twice $\lambda_{\mathrm{sc}}$. While a microstructural evaluation is not required for application of eqn (9), the impedance measurement must yield both the 
capacitance and resistance of both bulk and grain boundary responses. This expression and the values of $C_{\mathrm{GI}}$ and $C_{\mathrm{GB}}$ obtained at each temperature have been used to determine the specific grain boundary conductivity presented in Fig. 3(a).

The extent of carrier concentration depletion (or enhancement) in the space-charge region depends on the electrical potential profile within the space-charge layer. Under the assumption that the dopant concentration is position-independent (the flat-profile approximation) the solution to the Poisson equation yields the familiar Mott-Schottky electric potential profile ${ }^{36}$

$$
\Delta \phi(x)=\Delta \phi_{\mathrm{sc}}\left(1-\frac{x}{\lambda_{\mathrm{sc}}}\right)^{2}
$$

where $\Delta \phi(x)$ is the potential difference between the value at position $x$ from the grain boundary interface and the value in the bulk (infinitely far from the interface), and the space-charge layer width is given by

$$
\lambda_{\mathrm{sc}}=\sqrt{\frac{2 \varepsilon_{r} \varepsilon_{0} \Delta \phi_{\mathrm{sc}}}{e\left[\mathrm{Sm}_{\mathrm{Ce}}\right]}}
$$

where $\varepsilon_{r}$ is the relative dielectric constant, $\varepsilon_{0}$ is the vacuum permittivity, and $e$ is the electron charge. In the dilute-solution limit and under equilibrium conditions, it can be shown that the carrier concentration within the space-charge region is, in turn, given by

$$
c_{i}(x)=c_{\infty, i} \exp \left(-\frac{z_{i} e \Delta \phi(x)}{k_{\mathrm{B}} T}\right)
$$

where $c_{\infty, i}$ indicates the concentration of species $i$ in the bulk, $z_{i}$ is the valence of species $i$, and $k_{\mathrm{B}}, T$, and are the Boltzmann constant and temperature respectively. Integration of eqn (12) across the extent of the space-charge layer yields the total carrier concentration within the space charge region. With that result and the assumption of position-independent mobility the partial conductivity in the space-charge layer is approximated as: ${ }^{37}$

$$
\sigma_{\mathrm{sc}, i}=\frac{\sigma_{\infty, i} 2 z_{i} e \Delta \phi_{\mathrm{sc}}}{k_{\mathrm{B}} T}\left(\exp \left(\frac{z_{i} e \Delta \phi_{\mathrm{sc}}}{k_{\mathrm{B}} T}\right)-1\right)^{-1}
$$

where $\sigma_{\infty, i}$ is the bulk conductivity of the species $i$. Thus, modifications to the carrier concentrations induced by the space space-charge potential will modify the partial conductivities in the space charge region.

The total grain boundary conductivity is simply the sum of the partial conductivities $\left(\sigma_{\mathrm{GB}}^{\mathrm{sp}}=\sum_{i} \sigma_{\mathrm{sc}, i}\right)$. Combining this expression with eqn (13) and (9) yields an expression for the space-charge potential in terms of the experimentally measured resistances and capacitances:

$$
\frac{C_{\mathrm{GI}}}{R_{\mathrm{GB}} C_{\mathrm{GB}}}=\frac{2 e \Delta \phi_{\mathrm{sc}}}{k_{\mathrm{B}} T} \sum_{i}\left[\frac{R_{\mathrm{GI}, i}}{z_{i}}\left(\exp \left(\frac{z_{i} e \Delta \phi_{\mathrm{sc}}}{k_{\mathrm{B}} T}\right)-1\right)\right]^{-1}
$$

Numerical solution of eqn (14) yields the space-charge potential from the measured bulk and grain boundary resistances and capacitances. In dry air, with oxygen vacancies as the sole carriers (with the bulk concentration fixed by doping), the computation is straightforward. ${ }^{37}$ Where more than one carrier is present, additional information regarding the bulk resistance of each of the carriers are required. In the present analysis, which is limited to oxidizing conditions, humidification introduces protons as potential additional carriers and these are treated by evaluating two limiting conditions, as described later in this section.

At this stage it is important to reconsider the treatment of the grain boundary core as transparent with respect to charge transport and the overall applicability of the space-charge model to the system under consideration here. From eqn (11) it is evident that the space-charge layer thickness decreases with dopant concentration and may even approach the thickness of the grain boundary core. In the present case, using eqn (8) and the measured mean grain size of $0.36 \mu \mathrm{m}$, we compute a grain boundary width $\left(2 \lambda_{\mathrm{sc}}\right)$ of $1.2 \mathrm{~nm}\left(250^{\circ} \mathrm{C}\right.$, dry air). While this result implies a space-charge width that is only two times the lattice constant of ceria, the distance is nevertheless several times greater than the ionic radii of the species of interest. Furthermore, whatever the properties of the core, the space-charge region extends beyond it (the two are additive in terms of their resistive contributions) and thus potentially comparable dimensions between the core and the space-charge region does not invalidate the existence of the latter. The assumption that the core is moreover ion-reversible is specifically motivated here by the observed increase in the grain boundary resistance upon humidification and invariance upon isotopic exchange, behaviors not expected for transport limited by the core itself. The validity of the assumption can furthermore be evaluated from a comparison of the grain boundary width implied directly from the impedance spectra according to eqn (8) and that implied from a solution to eqn (11). Although these two values cannot be computed entirely independently, agreement between the two terms is a necessary requirement for validation of the space-charge model in conjunction with an ion-reversible core. The two values are plotted in Fig. 7 as a function of temperature (where the two limiting cases discussed below are considered for the case of eqn (11)). Reasonable agreement is observed, for both the dry and hydrated cases, with thickness values ranging from 0.8 to $1.2 \mathrm{~nm}$. The agreement is best at the lowest temperature examined, $250{ }^{\circ} \mathrm{C}$, presumably due to the accuracy of the measured dielectric constants as a result of the accessibility of the entire impedance response of the bulk. Additional important support for the validity of the assumption of an ion-reversible grain boundary core for 1-20 at.\% gadolinia-doped ceria is provided by the work of Avila-Paredes et al. ${ }^{26}$

The space-charge potential determined here under dry air using eqn (14) is $0.193 \mathrm{~V}$ at $250{ }^{\circ} \mathrm{C}$, rising to $0.221 \mathrm{~V}$ at $350{ }^{\circ} \mathrm{C}$, Fig. 8(a), values typical for doped ceria. ${ }^{22,26,27}$ Upon humidification we consider the possibility of non-zero proton uptake in the bulk of the material. Because the experimental bulk conductivity is unchanged between humidified and dry atmospheres (Fig. 2(a) and 3(a)), two limits for the bulk uptake of protons in ceria are examined: either it is zero (the lower limit), or the uptake has a maximum value given by the minimum resolution in the impedance measurements (in combination with an estimate of the relative mobility of protons).

In the zero-uptake, lower limit, oxygen vacancies are the sole charge carrier species in both the space-charge layer and 


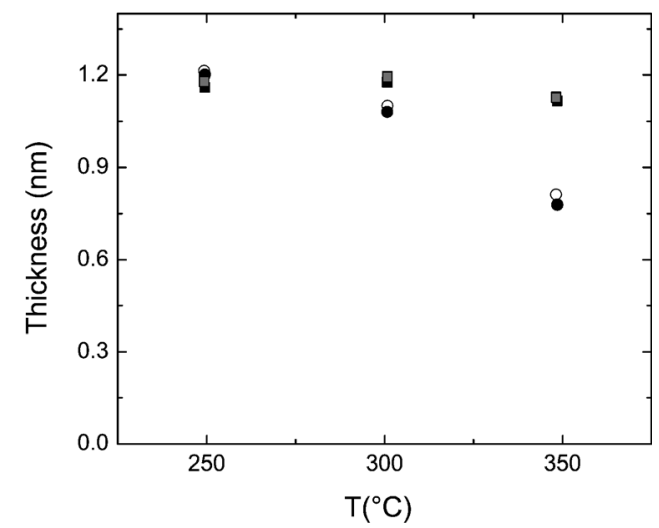

Fig. 7 Comparison of the space-charge layer thicknesses calculated using eqn (8), shown as circles $(\delta)$, and those calculated using eqn (11), shown as squares $\left(2 \lambda_{\mathrm{sc}}\right)$. Closed symbols are for dry conditions, open symbols are for humidified conditions $\left(p_{\mathrm{H}_{2} \mathrm{O}}=0.023 \mathrm{~atm}\right.$, white $=$ upper-bound limit for proton concentration and grey $=$ lower bound, zero uptake limit). $p_{\mathrm{O}_{2}}=0.21 \mathrm{~atm}$ for all measurements.

the bulk and, again, the space-charge potential can be readily computed. For the upper-bound limit, an estimate of the maximum bulk proton uptake is obtained by taking the maximum error in the resistance measurement to be $0.1 \%$ and taking the protons to have a mobility 100 times that of oxygen vacancies. ${ }^{33}$ These values imply a maximum bulk proton concentration upon humidification to be $2 \times 10^{-5}$ times that of the oxygen vacancy concentration (where the temperature dependence is ignored). With this estimate, it is possible to compute, using eqn (14), the upper-bound limit of the space-charge potential.

Both approaches, either assuming zero proton uptake or allowing for non-zero uptake, demonstrate that the increase in the grain boundary resistance upon humidification corresponds to an increase in the space-charge potential relative to the dry condition, Fig. 8(a). The increase is approximately constant, 5-6 $\mathrm{mV}$, over the temperature range examined, and is slightly higher if one allows for proton uptake. Changes in the spacecharge potential imply, by definition, changes in the extent of depletion of oxygen ion vacancies within the space-charge region. Using eqn (12), it is possible to compute the oxygen vacancy concentration at the core|space-charge layer interface $\left(\left[\mathrm{V}_{\mathrm{O}}^{\bullet \bullet}\right]_{0}\right.$ at $\left.x=0\right)$ for each of the three cases: dry, hydrated with zero bulk proton uptake, and hydrated with maximum bulk proton uptake. As shown in Fig. 8(b), in the zero-proton uptake limit, the vacancy concentration decreases by $24 \%$ at $250{ }^{\circ} \mathrm{C}$ upon hydration, whereas in the upper-bound limit, the decrease is $28 \%$. Thus, the approximately $0.5 \%$ difference in space-charge potentials computed for the two limits manifests itself as an approximately $4 \%$ difference in oxygen vacancy concentration. Simultaneous with the decrease in oxygen vacancy concentration, the partial resistance in the space-charge region due to oxygen vacancies, computed according to eqn (13), increases upon hydration, Fig. 8(c). The difference between the zero-uptake and upper-bound limits is again approximately $4 \%$, and, given the essentially identical space-charge layer widths, Fig. 7, the behavior reflects the decrease in oxygen vacancy carrier concentration as well as the fact that, in the upper-bound limit, a portion of the current is carried by protons.

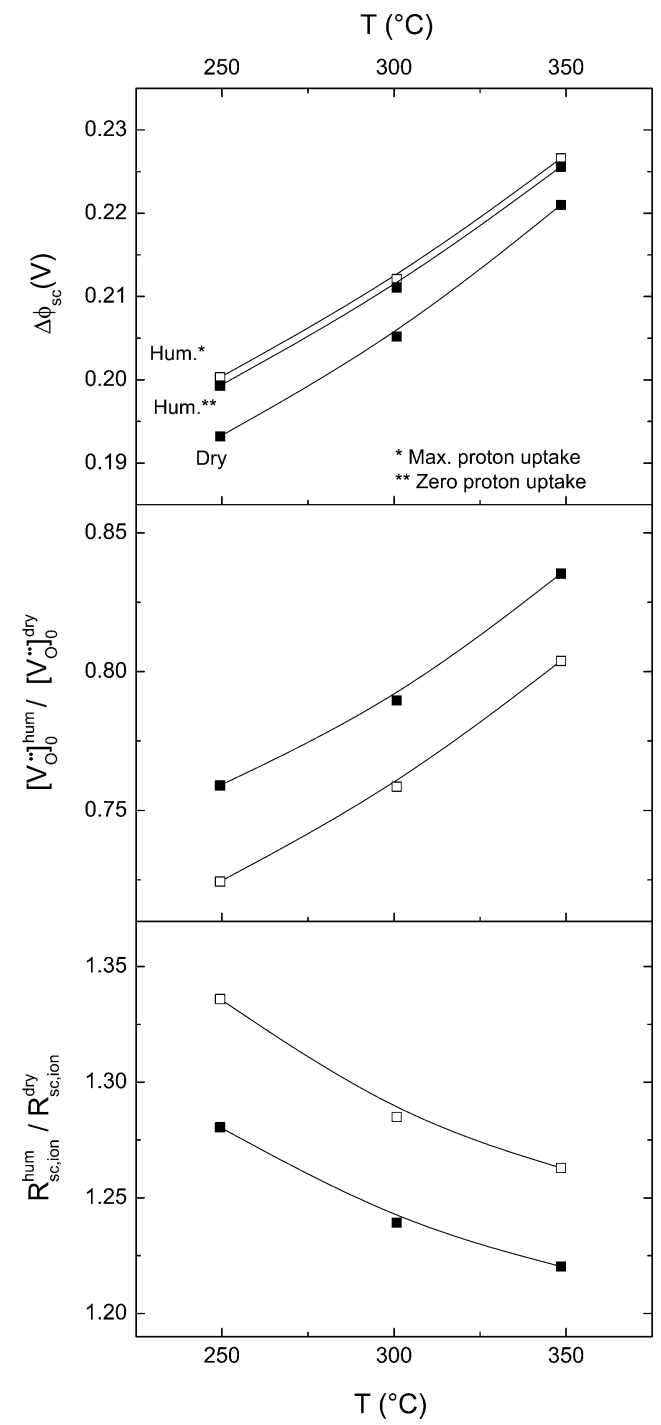

Fig. 8 Parameters computed from application of the spacecharge model under dry (closed squares) and humidified conditions $\left(p_{\mathrm{H}_{2} \mathrm{O}}=0.023 \mathrm{~atm}\right)$, for the maximum proton uptake limit (open squares) and zero uptake limit (grey squares): (a) Space-charge potential; (b) ratio of oxygen vacancy concentration at the core|spacecharge layer interface (denoted by subscript ' 0 ') under a humidified atmosphere to the value under a dry atmosphere; and (c) ratio of the oxygen vacancy partial resistance in space-charge layer (denoted by subscript 'sc') under humidified atmosphere to the value under dry atmosphere. $p_{\mathrm{O}_{2}}=0.21 \mathrm{~atm}$ for all measurements. Lines are guide to the eye.

It is of value to consider the maximum role of protons in the space-charge regions within the bounds of the upper limit defined here. Given the form of eqn (12), oxygen vacancy depletion is more severe than proton depletion because of the difference in valence of the two species. Accordingly, the concentration ratio, $\left[\mathrm{OH}_{\mathrm{O}}^{\bullet}\right] /\left[\mathrm{V}_{\mathrm{O}}^{\bullet \bullet}\right]$, at the core/space-charge layer interface is substantially higher than the same ratio in the bulk. Specifically, the former ranges from $1.3 \times 10^{-3}$ to $1.7 \times 10^{-3}$ with temperature (not shown), whereas the latter is taken to be fixed at $2 \times 10^{-5}$. Such an enhancement in proton concentration is reflected in the calculated proton transference number in the space-charge layer $\left(t_{\mathrm{sc}, \text { pon }}=\sigma_{\mathrm{sc}, \text { pon }} /\left(\sigma_{\mathrm{sc}, \text { pon }}+\sigma_{\mathrm{sc}, \text { ion }}\right)\right.$, 


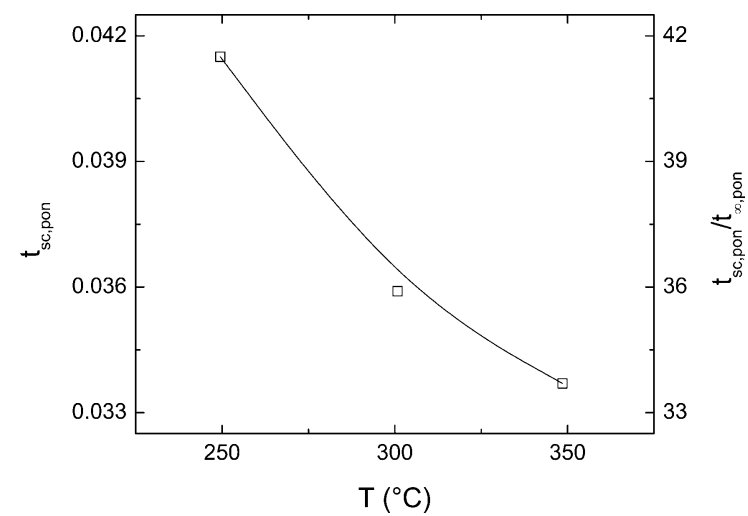

Fig. 9 Proton transference number in the space-charge layer (in the maximum proton uptake limit): (left axis) absolute value, and (right axis) relative to the bulk proton transference number ( $\sigma_{\infty, \text { pon }}=10^{-3} \sigma_{\infty \text {,ion }}$ assumed $)$. Line is a guide to the eye. $p_{\mathrm{H}_{2} \mathrm{O}}=0.023 \mathrm{~atm}$ and $p_{\mathrm{O}_{2}}=0.21 \mathrm{~atm}$ for all measurements.

Fig. 9, which is 42 times larger than that in the bulk $\left(250{ }^{\circ} \mathrm{C}\right)$. Despite such an enhancement, because the bulk proton conductivity is so low, the absolute grain boundary proton transference number is no higher than $4.2 \%$. This is consistent with the observation that $\mathrm{H}_{2} \mathrm{O}$-to- $\mathrm{D}_{2} \mathrm{O}$ isotopic exchange had a minimal impact on the observed grain boundary resistance. The actual proton transference number in the space-charge layer is likely to be lower as the true bulk proton uptake lies between the two limits examined here.

In sum, the analysis indicates that the space-charge potential increases on hydration, increasing the depletion of oxygen vacancies in the space-charge region relative to the dry condition. For non-zero bulk proton uptake on hydration, protons are also depleted in the space-charge region. While the depletion of oxygen vacancies is more significant than protons, the overall low proton concentrations prevent the proton from becoming a significant carrier. Accordingly, we observe an overall decrease in grain boundary conductivity upon hydration due to oxygen vacancy depletion without significant protonic contributions to the grain boundary conduction.

These results beg the question as to why the space-charge potential changes upon hydration. A positive $\Delta \phi_{\mathrm{sc}}$ implies that the total charge carried by cations in the grain boundary core exceeds that carried by anions. Hydration apparently increases this imbalance. While many scenarios can be considered that lead to such behavior, we propose that water molecules are incorporated into the grain boundary core in a fashion that enhances the energetic differences between oxide ion vacancy formation in the bulk and at the grain boundary interface. Specifically, if sites otherwise occupied by oxide ions in the core become occupied by neutral water molecules (or hydroxyl groups of less absolute negative charge than oxide ions), yet bonding considerations require the ratio of occupied anion and cation sites to remain constant, the effect would be exactly what is observed: an increase in the ratio of positive to negative charge. Uptake of water into the grain boundary core of oxides has been reported in a wide range of materials, ${ }^{41}$ and thus incorporation of water into the intergranular boundaries in samaria-doped ceria would not be unusual. In the scenario proposed, minimal differences between grain boundary properties under $\mathrm{H}_{2} \mathrm{O}$ and $\mathrm{D}_{2} \mathrm{O}$ are expected, as observed, and eventual saturation of absorption sites is also expected, consistent with the data in Fig. 4.

\section{Conclusions}

We have evaluated the transport properties of acceptor doped, microcrystalline ceria that is free of apparent grain boundary impurities under a range of atmospheres. Upon humidification under oxidizing conditions the grain boundary resistance increases measurably, whereas the bulk properties are unchanged. The effect is insensitive to $\mathrm{H}_{2} \mathrm{O}$-to- $\mathrm{D}_{2} \mathrm{O}$ isotopic exchange. These observations indicate that neither the grain boundary core nor intergranular phases are the source of the resistance. Accordingly, the behavior is interpreted in terms of the space-charge model. The analysis suggests that the space-charge potential increases upon hydration, inducing depletion of oxygen vacancies in the space-charge region. While the effect is relatively small at temperatures above $450{ }^{\circ} \mathrm{C}$, presumably due to low water solubility in the grain boundary core at high temperatures, the increase in space-charge layer resistance upon hydration can exceed $20 \%$ under moderate conditions (e.g., $22 \%$ at $250{ }^{\circ} \mathrm{C}$ under $\left.p_{\mathrm{H}_{2} \mathrm{O}}=0.023 \mathrm{~atm}\right)$. The atypical observation that the grain boundary resistance of microcrystalline SDC increases upon hydration is attributed to two key factors: the large grain size, which renders proton conduction in the direction parallel to the grain boundary negligible, and the low bulk proton solubility, which keeps the proton transference number within the space-charge layer low, despite its likely enhancement over the bulk value. The analysis further demonstrates that one can neglect protons within the space-charge layer as the material inherently displays extremely low bulk proton uptake and conductivity. The situation is dramatically different from nanocrystalline materials, in which proton transport via the grain boundary core in a direction parallel to that of current flow can become dominant at low temperatures. While one cannot entirely rule out the possibility that the increases in grain boundary resistance may be directly due to changes in the structure of the grain boundary core, all of the experimental features observed can be described using the space-charge model.

\section{Acknowledgements}

We gratefully acknowledge the financial support of the National Science Foundation, Grant No. DMR-0604004. Additional support was provided by the National Science Foundation through the Caltech Center for the Science and Engineering of Materials, a Materials Research Science and Engineering Center (DMR-052056).

\section{References}

1 A. Trovarelli, Catal. Rev., 1996, 38, 439-520.

2 K. Eguchi, T. Setoguchi, T. Inoue and H. Arai, Solid State Ionics, 1992, 52, 165-172.

3 M. Mogensen, N. M. Sammes and G. A. Tompsett, Solid State Ionics, 2000, 129, 63-94.

4 W. C. Chueh, W. Lai and S. M. Haile, Solid State Ionics, 2008, 179, 1036-1041.

5 W. Lai and S. M. Haile, J. Am. Ceram. Soc., 2005, 88, 2979-2997. 
6 T. Nakamura, T. Kobayashi, K. Yashiro, Atsushi, T. Otake, K. Sato, J. Mizusaki and T. Kawada, J. Electrochem. Soc., 2008, 155, 563-569.

7 W. C. Chueh and S. M. Haile, ChemSusChem, 2008, 2, 735-739.

8 H. Kaneko, T. Miura, H. Ishihara, S. Taku, T. Yokoyama, H. Nakajima and Y. Tamaura, Energy, 2007, 32, 656-663.

9 N. Sakai, K. Yamaji, T. Horita, H. Kishimoto, Y. P. Xiong and H. Yokokawa, Solid State Ionics, 2003, 175, 387-391.

10 N. Sakai, K. Yamaji, T. Horita, H. Yokokawa, Y. Hirata, S. Sameshima, Y. Nigara and J. Mizusaki, Solid State Ionics, 1999, 125, 325.

11 M. Shirpour, G. Gregori, R. Merkle and J. Maier, Phys. Chem. Chem. Phys., 2011, 13, 937-940.

12 E. Ruiz-Trejo and J. Kilner, J. Appl. Electrochem., 2009, 39, 523-528.

13 H. Takamura and N. Takahashi, Solid State Ionics, 2010, 181, 100, DOI: $10.1016 /$ j.ssi.2009.04.017.

14 S. Kim, U. Anselmi-Tamburini, H. J. Park, M. Martin and Z. A. Munir, Adv. Mater., 2008, 20, 556-559.

15 S. Kim, R. Merkle and J. Maier, Solid State Ionics, 2003, 161, $113-119$.

16 S. Kim, H. J. Avila-Paredes, S. Wang, C.-T. Chen, R. A. D. Souza, M. Martin and Z. A. Munir, Phys. Chem. Chem. Phys., 2009, 11, 3035-3038.

17 G. Chiodelli, F. Maglia, U. Anselmi-Tamburini and Z. A. Munir, Solid State Ionics, 2009, 180, 297-301.

18 K. D. Kreuer, Solid State Ionics, 1999, 125, 285-302.

19 K. D. Kreuer, W. Munch, M. Ise, T. He, A. Fuchs, U. Traub and J. Maier, Ber. Bunsenges. Phys. Chem., 1997, 101, 1344-1350.

20 H. L. Tuller and A. S. Nowick, J. Phys. Chem. Solids, 1977, 38 859-867.

21 W. C. Chueh and S. M. Haile, Phys. Chem. Phys. Chem., 2009, 11, 8144-8148.

22 W. Lai, Thesis, California Institute of Technology, 2007.
23 A. Jasper, J. A. Kilner and D. W. McComb, Solid State Ionics, 2008, 179, 904-908.

24 R. Gerhardt and A. S. Nowick, J. Am. Ceram. Soc., 1986, 69, 641-646.

25 T. S. Zhang, J. Ma, S. H. Chan, P. Hing and J. A. Kilner, Solid State Sci., 2004, 6, 565-572.

26 H. J. Avila-Paredes, K. Choi, C.-T. Chen and S. Kim, J. Mater. Chem., 2009, 19, 4837-4842.

27 X. Guo, W. Sigle and J. Maier, J. Am. Ceram. Soc., 2003, 86, $77-87$.

28 X. Guo and R. Waser, Prog. Mater. Sci., 2006, 51, 151-210.

29 A. S. Nowick, A. V. Vaysleyb and I. Kuskovsky, Phys. Rev. B, 1998, 58, 8398-8406.

30 H. Yamamura, S. Takeda and K. Kakinuma, J. Ceram. Soc. Jpn., 2007, 115, 264-268

31 S. M. Haile, D. L. West and J. Campbell, J. Mater. Res., 1998, 13, $1576-1595$.

32 T. Norby and Y. Larring, Curr. Opin. Solid State Mater. Sci., 1997, 2, 593-599.

33 K. D. Kreuer, E. Schonherr and J. Maier, Solid State Ionics, 1994 70-71, 278-284.

34 J. Maier, Physical Chemistry of Ionic Materials, Wiley, 2004.

35 J. Frenkel, Kinetic Theory of Liquids, Oxford University Press, Oxford, 1946.

36 S. Kim, J. Fleig and J. Maier, Phys. Chem. Chem. Phys., 2003, 5, $2268-2273$.

37 X. Guo and J. Maier, J. Electrochem. Soc., 2001, 148, E121-E126. 38 S. Kim and J. Maier, J. Electrochem. Soc., 2002, 149, J73-J83.

39 A. Tschope, J. Electroceram., 2004, 14, 5-23.

40 A. Tschope, E. Sommer and R. Birringer, Solid State Ionics, 2000, 139, 255-265.

41 H. J. Park, C. Kwak, K. H. Lee, S. M. Lee and E. S. Lee, J. Eur. Ceram. Soc., 2009, 29, 2429-2437. 